\title{
Unsteady Flow past a Combined Pitching and Plunging Aerofoil using an Implicit RANS Solver
}

\author{
K. Siva Kumar \\ Computational and Theoretical Fluid Dynamics \\ Division, Council of Scientific and Industrial Research- \\ National Aerospace Laboratories, \\ Bangalore, Karnataka-560017, INDIA \\ email id:shivak@ctfd.cmmacs.ernet.in
}

\begin{abstract}
Unsteady Reynolds-averaged Navier-Stokes (RANS) computations are presented for low Mach number flow past a combined pitching and plunging NACA 0012 aerofoil. The Implicit RANS solver used for obtaining timeaccurate solutions is based on a finite volume nodal point spatial discretization scheme with dual time stepping. The aim is to validate the unsteady solver for flapping motion of the aerofoil. Results are presented in the form of aerodynamic coefficients and compared with available literature, thus demonstrating the capability of the solver to provide useful unsteady input data for aeroelastic and aeroacoustic analysis.
\end{abstract}

Keywords- unsteady flow, RANS solver, implicit method, dual time stepping, flapping aerofoil.

\section{INTRODUCTION}

Unsteady flows are encountered in many aerospace applications and prediction of unsteady air loads plays a vital role in aircraft and helicopter design [1-3]. Since wind tunnel testing of unsteady flow situations is difficult and expensive, computational studies of wing stall, dynamic stall, bladevortex interaction of helicopter rotors and aeroelastic problems like flutter, buffeting and gust- response etc., can provide important design data.

Flying birds usually flap their wings to generate both lift and thrust. Flapping motion of birds has a coupled pitching and plunging oscillation with some phase difference between them. Recent experimental and computational studies investigated the kinematics, dynamics, flow characteristics of flapping wings and shed some light on the lift, drag, and propulsive power considerations [4, 5]. Yang et al. [6] have computed a sinusoidally pitching and plunging NACA 0012 aerofoil in a uniform stream of low speeds for different motion parameters by using inviscid version of a threedimensional unsteady compressible Euler/Navier-Stokes flow solver and optimized for high propulsive efficiency and for high time-averaged thrust coefficient. Theodorsen [7] has developed compact expressions for forces and moments of a flapping plate aerofoil for small perturbed inviscid and incompressible flow. In the prediction of unsteady pressure distributions over aerofoils, the steady-state Kutta-

\author{
Sharanappa V. Sajjan \\ Computational and Theoretical Fluid Dynamic Division, \\ Council of Scientific and Industrial Research-National \\ Aerospace Laboratories, \\ Bangalore, Karnataka-560017, INDIA \\ email id: svsajjan@ctfd.cmmacs.ernet.in
}

Joukowsky condition is assumed. The flow is treated in two classes: the non circulating flow due to the aerofoil vertical acceleration and the circulatory flow due to the wake vortices. Many important features of flapping aerofoil behavior are depicted by the classical linear theory. The thrust force experienced by the flapping aerofoil was given by Garrick [8]. Tuncer and Platzer [9] used a compressible Navier-Stokes solver to compute the unsteady turbulent flow fields and obtained high propulsive efficiency when the flow remains mostly attached over the aerofoil oscillated in plunge and pitch. Isogai et al. [10] performed Navier-Stokes simulations of flow over a NACA 0012 aerofoil undergoing combined pitching and plunging motion at $R e=10^{5}$. Ramamurti and Sandberg [11] performed numerical simulation of the flow over a flapping NACA 0012 aerofoil using a finite element incompressible Navier-Stokes solver at a Reynolds number of 1100 . They found that the critical parameter which affects the thrust generation is $k h$ rather than $k$. They also found that maximum thrust is obtained when the pitching motion leads the plunging motion by $120^{\circ}$ and the maximum propulsive efficiency occurs at $\varnothing=90^{\circ}$. Anderson et al. [12] measured the time-averaged thrust coefficient, input power coefficient, and propulsion efficiency of a NACA 0012 aerofoil undergoing combined sinusoidal plunging and pitching motion in the testing tank facility at MIT.

\section{IMPRANS SOLVER}

The two-dimensional Reynolds-averaged Navier-Stokes equations for a moving domain can be written in nondimensional conservative form as

$$
\frac{\partial U}{\partial t}+\frac{\partial F}{\partial x}+\frac{\partial G}{\partial y}=\frac{\partial V}{\partial x}+\frac{\partial W}{\partial y}
$$

where

$$
U=\left[\begin{array}{l}
\rho \\
\rho u \\
\rho v \\
e
\end{array}\right], F=\left[\begin{array}{c}
\rho\left(u-x_{t}\right) \\
\rho u\left(u-x_{t}\right)+p \\
\rho v\left(u-x_{t}\right) \\
e\left(u-x_{t}\right)+p u
\end{array}\right], G=\left[\begin{array}{c}
\rho\left(v-y_{t}\right) \\
\rho u\left(v-y_{t}\right) \\
\rho v\left(v-y_{t}\right)+p \\
e\left(v-y_{t}\right)+p v
\end{array}\right]
$$




$$
\begin{aligned}
& V=V_{1}\left(U, U_{x}\right)+V_{2}\left(U, U_{y}\right)
\end{aligned}
$$

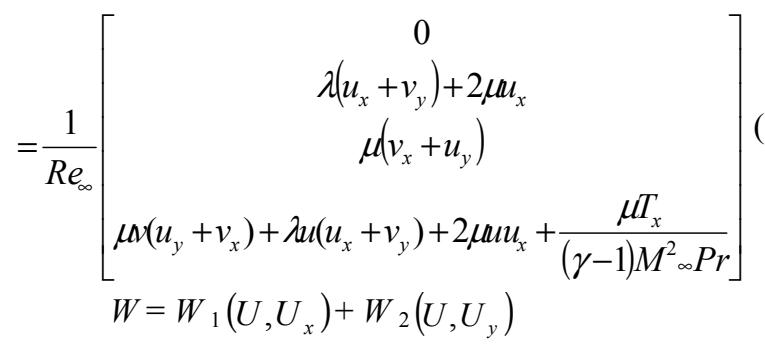

$$
\begin{aligned}
& =\frac{1}{R e_{\infty}}\left[\begin{array}{c}
0 \\
\mu\left(u_{y}+v_{x}\right) \\
\lambda\left(u_{x}+v_{y}\right)+2 \mu v_{y} \\
\mu u\left(u_{y}+v_{x}\right)+\lambda\left(u_{x}+v_{y}\right)+2 \mu \omega v_{y}+\frac{\mu T_{y}}{(\gamma-1) M_{\infty}^{2} P r}
\end{array}\right]
\end{aligned}
$$

Here $x$ and $y$ are the Cartesian coordinates and $t$ is the time variable; $x_{\mathrm{t}}$ and $y_{t}$ are the Cartesian velocity components of the moving domain. For a fixed domain, the grid speeds $x_{t}$ and $y_{t}$ are zero. $U$ is the vector of conserved variables; $F, G$ are inviscid flux vectors and $V, W$ are viscous flux vectors. The primitive variables are density $\rho$, velocity components $u, v$ in the $x$ and $y$ directions, pressure $p$, temperature $T$ and total energy $e$ per unit volume. The non-dimensional variables used in the above equations have been obtained by using the following free stream values as reference quantities: $\rho_{\infty}$ (density), $U_{\infty}$ (velocity), $\mu_{\infty}$ (viscosity), $\rho_{\infty} U_{\infty}^{2}$ (pressure), $T_{\infty}$ (temperature), and so on. Some characteristic length such as chord $c$ of an aerofoil is chosen as the length scale.

$M_{\infty}$ and $R e_{\infty}$ are the free stream Mach number and Reynolds number respectively; $\gamma$ is the ratio of specific heats and $P r$ is the Prandtl number. In addition, the viscosity coefficients $\lambda$ and $\mu$ given by the Stokes relation

$$
3 \lambda+2 \mu=0
$$

and the Sutherland's law of viscosity

$$
\mu=C_{1}\left[\frac{T^{3 / 2}}{T+C_{2}}\right]
$$

For turbulent flows, the laminar viscosity coefficient $\mu$ is replaced by $\mu+\mu_{t}$, and $\mu / P r$ is replaced by $\mu / P r+\mu_{t} /$ $\operatorname{Pr}_{t}$; the turbulent viscosity coefficient $\mu_{t}$ and the turbulent Prandtl number $\operatorname{Pr}_{t}$ are provided by a turbulence model. Finally the system is closed using the perfect gas equation of state in non-dimensional form as

$$
P=\frac{\rho T}{\not M^{2}}
$$

The Euler equations for inviscid flow are obtained from the Navier-Stokes equations by setting

$$
\frac{1}{R e_{\infty}}=0
$$

\section{COMPUTATIONAL METHOD}

Applying Euler's implicit time differencing formula [13]

$$
U^{n}=U^{n+1}-\left(\frac{\partial U}{\partial t}\right)^{n+1} \Delta t+O\left(\Delta t^{2}\right)
$$

to the governing Eq. (1), we obtain

$$
\Delta U^{n}+\Delta t\left[\frac{\partial}{\partial x}(F-V)+\frac{\partial}{\partial y}(G-W)\right]^{n+1}=0
$$

Here $U^{n}=U(t)=U(n \Delta t)$ is the solution vector at time level $n$ and $\Delta U^{n}=\left(U^{n+1}-U^{n}\right)$ is the change in $U^{n}$ over time step $\Delta t$. In order to facilitate the finite volume formulation, the above equations are written in the integral form as

$$
\iint_{\Omega} \Delta U^{n} d x d y+\Delta t \int_{\Gamma}\left[(F-V)^{n+1} d y-(G-W)^{n+1} d x\right]=0
$$

where $\Omega$ is any two-dimensional flow domain and $\Gamma$ is the boundary curve.

In the nodal point finite volume approach $[14,15]$, the flow variables are associated with each mesh point of the grid and the integral conservative equations are applied to each control volume obtained by joining the centroids of the four neighbouring cells of a nodal point. Application of nodal point spatial discretization to Eq. (10). leads to the following equations for the computational cell $\Omega_{i j}$

$$
\Delta U_{i j}^{n} h_{i j}+\Delta t \int_{\Gamma_{i j}}\left[(F-V)^{n+1} d y-(G-W)^{n+1} d x\right]=0
$$

Linearzing the changes in flux vectors using Taylor's series expansions in time and assuming locally constant transport properties, Eq. (11). can be simplified to

$$
\begin{aligned}
& \Delta U_{i j}^{n}+\frac{\Delta t}{h_{i j}}\left[\int_{\Gamma_{i j}}\left\{A^{n}-\frac{\partial}{\partial x} R^{n}\right\} \Delta U^{n} d y-\int_{\Gamma_{i j}}\left\{B^{n}-\frac{\partial}{\partial y} S^{n}\right\} \Delta U^{n} d x\right] \\
& =-\frac{\Delta t}{h_{i j}}\left[\int_{\Gamma_{i j}}(F-V)^{n} d y+\int_{\Gamma_{i j}}(G-W)^{n} d x\right]
\end{aligned}
$$

Here $A, B, R$ and $S$ are the Jacobian matrices which are given by

$$
A=\frac{\partial F}{\partial U}, B=\frac{\partial G}{\partial U}, R=\frac{\partial V_{1}}{\partial U_{x}} \text { and } S=\frac{\partial W_{2}}{\partial U_{y}}
$$

This RANS solver has been extensively validated for computing unsteady flow past pitching aerofoils and wings [16], helicopter rotor blades [17, 18], wind turbines [19] etc. 
Here, the solver has been applied for computing twodimensional unsteady compressible viscous flow over combined pitching and plunging NACA 0012 aerofoil.

\section{GRID GENERATION}

For all present computations, the structured C-type grid, of size $247 \times 65$ (stream-wise $\times$ normal) moving with combined pitching and plunging NACA 0012 aerofoil is used which is shown in Fig. 2(a). 167 of the 247 points are distributed on the aerofoil surface and 41 points in the wake region. The outer boundary is located 30 chords away from the aerofoil surface. The first grid spacing on the aerofoil surface of $1.0 \times 10^{-5} \mathrm{c}$ was used in the direction normal to the aerofoil surface. The grid points are properly clustered near the leading, trailing edges and wall normal direction. The close-up view of the grid is shown in Fig. 2(b).

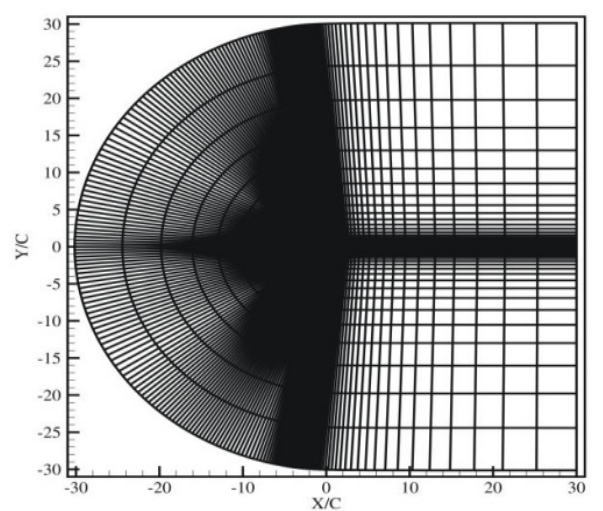

Fig. 2 (a). C- Grid around the NACA 0012

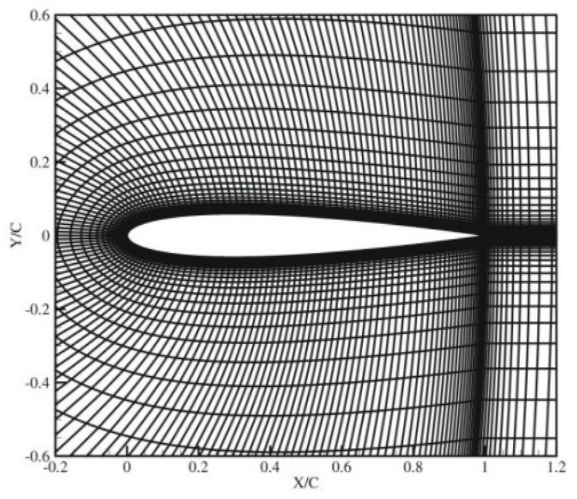

Fig. 2 (b). Close-up view of the aerofoil grid V. FLAPPING MOTION OF THE AEROFOIL

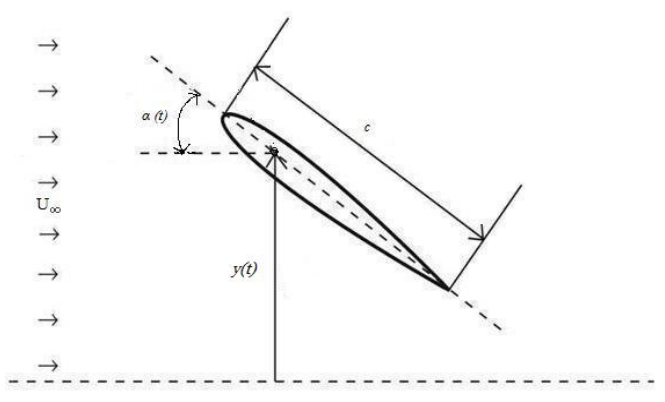

Fig. 3 (a). Aerofoil in combined pitching and plunging motion The sinusoidal motion of combined pitching and plunging aerofoil is defined by the following expressions. The plunging motion of an aerofoil is

$$
y(t)=h \sin (\omega t)
$$

where $t$ is physical time, $\omega$ and $h$ are the angular frequency and the amplitude of the plunging oscillation respectively, $h$ is positive in the upward direction. The non-dimensional time, $\tau=U_{\infty} t / c$, non-dimensional amplitude in plunge, $h_{a}=$ $h / c$, and the reduced frequency, $k=\omega c / 2 U_{\infty}$. Then the instantaneous non-dimensional plunging velocity of the aerofoil is given by

$$
y / U_{\infty}=2 k h_{a} \cos (2 k \tau)
$$

The coupled pitching oscillation is defined as rotating about a pivot point on the aerofoil chord which is shown in Fig. 3 (a). The instantaneous angle measured clockwise from the mean chord is $\alpha(t)$ which is given by

$$
\alpha(t)=\alpha_{m}+\alpha_{o} \sin (\omega t+\varnothing)
$$

The instantaneous non-dimensional pitching velocity of the aerofoil is given by

$$
\alpha / U_{\infty}=2 k \alpha_{o} \cos (2 k \tau+\varnothing)
$$

where $\alpha_{o}$ is the amplitude of pitching oscillation, $\alpha_{m}$ is the mean angle of attack and $\varnothing$ is the phase angle ahead of the plunging motion which is shown in Fig. 3 (b).

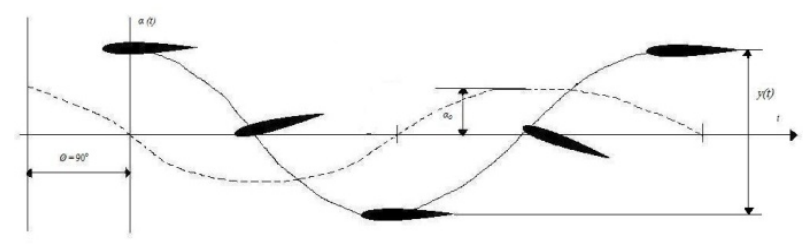

Fig. 3 (b). Aerofoil in combined pitching and plunging motion with a phase difference $\varnothing=90^{\circ}$

\section{RESULTS AND DISCUSSION}

The computations have been carried out for twodimensional unsteady viscous flow over a combined pitching and plunging aerofoil at low Mach number. For all simulations, steady state solutions are first obtained. After steady state convergence is reached, the aerofoil then undergoes a prescribed sinusoidal motion, both pitching about half chord and plunging motion. Five consecutive 
cycles were computed to obtain periodic solutions. The computational time step used was $\Delta t=0.005$ in all computations. Computations have been carried out for $\quad 0^{\circ}$ and $6^{\circ}$ mean angles of incidence with $M_{\infty}=0.1, \quad R e_{\infty}=$ $2.41 \times 10^{6}, k=0.27$, pitch amplitude of $30^{\circ}$, non-dimensional plunge amplitude of 1.25 and with a leading phase angle of $90^{\circ}$ between pitching and plunging motion.

Figure 4(a). and Figure 5(a). represents the instantaneous lift, pitching moment and thrust coefficient versus $y / c$ for a pitching-plunging NACA 0012 aerofoil computed by the IMPRANS Solver at $0^{\circ}$ mean angle of attack. Similarly for $6^{\circ}$ mean angle of attack the results are shown in Fig. 4(b). and Fig. 5(b). respectively. In both the cases the computed results are in reasonably good agreement with Euler solutions of Yang et al. [6]. The computed loops of the aerodynamic coefficients clearly demonstrate the hysteretic property existing between the up-stroke and down-stroke. The lift and the pitching moment values are higher during down stroke than during up stroke. The thrust values are smaller during the first half of up stroke compared to the second half of down stroke and become higher during the second half of up stroke than during the first half of down stroke. It can be seen from the figures that the concept entirely changes when the mean angle of attack is non-zero. The original two loops in a cycle for $C_{t}$ become one loop for a cycle. Higher thrust occurs during down stroke than during upstroke. The difference in predicted values and the values of Euler solutions of Yang et al. [6] is probably due to the presence of viscous effect. The aerodynamic loads have attained a periodic behavior after running four cycles of oscillations.

\section{CONCLUDING REMARKS}

The lift, pitching moment and thrust coefficients of a coupled pitching and plunging NACA 0012 aerofoil computed by using the Implicit Reynolds-averaged NavierStokes solver agrees well with the available literature, thus demonstrating the capability of the solver to provide useful unsteady input data for aeroelastic and aeroacoustic analysis.

\section{ACKNOWLEDGMENTS}

The authors gratefully acknowledge Dr. Vimala Dutta and Dr. P. K. Dutta for their constant encouragement and support during the work.

\section{REFERENCES}

[1] D. G. Mabey, 1999. Unsteady Aero-dynamics: Restrospect and prospect, Aero-nautical Journal, Vol. 103, No. 1019, Review Paper No. 003, 1 - 18 .

[2] W. J. McCroskey, 1982. Unsteady airfoils, Ann. Rev. Fluid Mech., Vol. 14, 285 - 311.

[3] W. J. McCroskey, 1988. Some Rotorcraft Applications of Computational Fluid Dynamics, NASA TM 100066.
[4] W. Shyy, M. Berg and D. Lyungvist,, 1999. Flapping and Flexible Wings for Biological and Micro Air Vehicles, Progress in Aerospace Sciences, Vol. 35, No. 5, pp. 455 - 505.

[5] T. J. Mueller(ed.), 2001. Fixed and Flapping Wing Aerodynamics for Micro Air Vehicles, Progress in Aeronautics and Astronautics, AIAA, Reston, VA, Vol. 195.

[6] S. Yang, S. Luo and F. Liu, 2006. Optimization of Unstalled Pitching and Plunging Motion of an Airfoil, AIAA paper, submitted to $44^{\text {th }}$ AIAA Aerospace Sciences Meeting and Exhibit, Reno, Nevada, 1055.

[7] T. Theodorsen, 1934. General theory of aerodynamic instability and the mechanism of flutter, NACA REPORT No. 496.

[8] I. E. Garrick, 1936. Propulsion of a flapping and oscillating airfoil, NACA Report No. 567.

[9] I. H. Tuncer and M. F. Platzer, 2000. Computational study of flapping airfoil Aerodynamics, Journal of Aircraft, Vol. 37, pp. 514 520

[10] K. Isogai, Y. Shinmoto and Y Watanabe, 1999. Effects of Dynamic Stall on Propulsive Efficiency and Thrust of Flapping Airfoil, AIAA Journal, Vol. 37, pp. 1145 - 1151.

[11] Ramamurti, R. and Sandberg, W., 2001. Simulation of Flow about Flapping Airfoils using Finite Element Incompressible Flow Solver, AIAA Journal, Vol. 39, pp. 253 - 260.

[12] J. M. Anderson, K. Streitlien, D. S. Barrett and M. S.Triantafyllou, 1998. Oscillating foils of high propulsive efficiency, J. Fluid Mech., 360, pp. 41 - 72.

[13] R. M. Beam and R. F. Warming, 1978. An Implicit Factored Scheme for the Compressible Navier-Stokes Equations, AIAA Journal, Vol. 16, No. 4, pp. 393 - 402.

[14] M. G. Hall, 1985. Cell Vertex Multi-grid Scheme for Solution of the Euler Equations RAE-TM-Aero 2029, Proc. Conf. on Numerical methods for fluid dynamics, pp. 303 - 345.

[15] A. Jameson, W. Schmidt and E. Turkel, 1981. Numerical Solution of Euler Equations by Finite Volume Methods Using Runge Kutta Time Stepping Schemes, AIAA Paper 81 - 1259.

[16] Sharanappa V. Sajjan, Vimala Dutta and P. K. Dutta 2008. Numerical Simulation of flow over pitching bodies using an implicit Reynoldsaveraged Navier-Stokes solver, Proc. of $12^{\text {th }}$ Asian congress of Fluid Mechanics, Daejeon, Korea.

[17] Vimala Dutta, Sharanappa and P. K. Dutta, 2005. Navier-Stokes Computations for a Helicopter Rotor Blade in Hover, Proc. Eighth Annual CFD Symposium, CFD Division of Aeronautical Society of India, Bangalore, CP 18.

[18] Sharanappa V. Sajjan, Vimala Dutta, and P. K. Dutta, 2006. Viscous Unsteady Flow around a Helicopter Rotor Blade in Forward Flight. Proc. $9^{\text {th }}$ Annual CFD symposium, CFD Division of Aeronautical Society of India, Bangalore.

[19] P. K. Dutta, Vimala Dutta, and Sharanappa V. Sajjan., 2007. RANS Computation of Flow past Wind Turbine Blades. Proc. of $7^{\text {th }}$ Asian Computational Fluid Dynamics Conference, Bangalore (Invited Paper). 

